\title{
The appropriate use of human albumin in a Brazilian University Hospital: therapeutic indication and dosage regimen
}

\author{
Tatiane Sayuri Ishida*1, Maria Cristina Sakai', Daniela Oliveira de Melo² \\ ${ }^{1}$ University Hospital of University of Sao Paulo, Pharmacy Department, Sao Paulo, SP, Brazil, ${ }^{2}$ Federal University of Sao \\ Paulo, Pharmaceutical Sciences Department, Diadema, SP, Brazil
}

\begin{abstract}
The objective of the study was to evaluate the use of human albumin in a Brazilian university hospital, in compliance with the institutional protocol and other clinical guidelines, taking into account the therapeutic indications and the dosage regimens. Data was obtained from the pharmacy dispensing records of human albumin, the requests for use it and, when available, the patient's records between January and October 2017. After evaluation the therapeutic indications and the dosage regimens were classified as "appropriate" and "inappropriate". The analysis of 98 requests showed that, when compared to the institutional protocol, $54(55.1 \%)$ requests had an inappropriate therapeutic indication. However, when a comparison was made between 25 medical records (54 requests) and other clinical guidelines, it was observed that the therapeutic indication had none classified as inappropriate. In addition, 16 (29.7\%) requests were considered inappropriate in relation to dosage regimens. From these results, it was possible to conclude that although the use of human albumin in the hospital was associated to a clinical protocol, it was outdated. Thus, it is possible to affirm that not only the adoption of a clinical protocol, but its periodical updating is an important strategy to promote the rational use of drugs.
\end{abstract}

Keywords: Albumins/drug utilization review. Health services. University Hospital/Brazil. Drugs/use.

\section{INTRODUCTION}

Human albumin (HA) is the major serum protein, accounting for about $50 \%$ of the total plasma circulating proteins. It is also the main responsible for maintaining intravascular oncotic pressure, accounting for at least $70 \%$ of it in healthy individuals (Valerio et al., 2016; Bernardi, Ricci, Zaccherini, 2014).

HA is a pharmaceutical product available in hyperosmotic concentrations (20 to $25 \%$ ) for reestablishing oncotic pressure and iso-osmotic concentration (5\%) for plasma volume replacement (Matos, Rozenfeld, 2005). Because HA is a blood derivative, it has limited availability and a manufacturing process with strict health and safety regulations. It is considered a safe product, although the potential risk of micro-organisms transmission or severe allergenic effects cannot be ruled out. As a consequence of these factors, HA is a product that has a high cost (Matos, Rozenfeld, 2005; Vaglio et

\footnotetext{
*Corresponding author: T. S. Ishida. Departamento de Farmácia, Hospital Universitário, Universidade de São Paulo. Av. Prof. Lineu Prestes, 2565, 05508-000 - São Paulo, SP, Brasil. Tel.: (55 11) 3091-9331. E-mail: tatiane.ishida@usp.br
}

al., 2014; Liumbruno et al., 2009; Casuccio et al., 2015; Corrêa et al., 2015).

The question of the rational use of HA in clinical hospital practice is still discussed today. This is because it is categorized as an A item in ABC analysis of most hospitals, compromising a significant part of hospital budgets. In addition, the recommendations for the use of HA are still very divergent, mainly because most of its therapeutic indications are related to physiological properties rather than scientific evidence of superior effectiveness to other therapeutic alternatives. In fact, for the introduction of HA into clinical practice, controlled studies have not been conducted to prove its effectiveness and safety for each of the therapeutic indications, as it occurs with other drugs (Matos, Rozenfeld, 2005; Casuccio et al., 2015; Caraceni, Tufoni, Bonavita, 2013; Borges Filho et al., 2010).

In 1975, the National Institute of Health (NIH) held a consensus conference to develop a protocol to rationalize the use of HA in the United States (Tullis 1977a; Tullis 1977b). From this consensus, countries such as Australia, Canada, Spain, France and Italy have also established committees and developed clinical practice guidelines 
or clinical protocols. Thus, the therapeutic indications of HA were classified as: 1) Approved indications, with convincing evidence; 2) Indications not approved, but with potential clinical benefit, most of the time with inconclusive evidence; 3) Indications not recommended (Casuccio et al., 2015; Vermeulen, Ratko, Erstad, 2010; Liumbruno et al., 2009; Stanford Health Care, 2017; Guy's and St. Thomas' NHS Foundation Trust, 2015; Caraceni et al., 2016; National Plasma Products Expert Advisory Group - Scotland NHS, 2016; Ontario Regional Blood Coordinating Network, 2012; Lyu et al., 2016).

Despite the establishment of these guidelines, the irrational use of HA is still very present in clinical practice. According to a European study published in 2013, up to $90 \%$ of HA prescriptions in clinical practice of several countries are not supported by clinical evidence, guidelines, or practical recommendations (Caraceni et al., 2013).

In Brazil, the national guidelines for the rational use of HA were established by the National Agency of Sanitary Surveillance (ANVISA) by Directors' Collegiate Resolution (RDC) No. 115, dated May 10, 2004 (Brasil, 2004). In this guideline, indications were classified into: 1) Formal indications; 2) Debatable indications; 3) Unsubstantiated indications. A study carried out in a quaternary hospital located in São Paulo city pointed out that of a total of 14,799 bottles of $20 \%$ human albumin consumed between January and October 2007, 4,191 (28\%) bottles were for unsubstantiated indications (Borges Filho et al., 2010). A similar result was found in a study conducted in a general hospital in the city of Rio de Janeiro, where the rate of the unsubstantiated indications represented $20.9 \%$ of the total prescriptions evaluated between March and June 2001 (Matos, Rozenfeld, 2005).

The economic repercussion of the irrational use of HA is also often pointed out in studies of the appropriate use of this medicine. According to a prospective observational study carried out in two university hospitals in Spain between October 1995 and March 1996, only $8.1 \%$ of HA prescriptions were considered appropriate. Therefore, there was an irrational use of 2,893 bottles of HA with a significant financial impact (74,306 ECU) (Vargas et al., 1997). The irrational use of HA in a quaternary hospital in São Paulo resulted in a loss of R\$ 1.36 million (Borges Filho et al., 2010).

Considering that the national guideline that orientate the use of HA was published in 2004, the high cost of the drug and its relevance in clinical practice, this study aims to evaluate the appropriateness of requests for albumin use (therapeutic indication and dosing regimen) in a hospital, according to the institutional protocol and other clinical guidelines published in the last years $(2007-2017)$.

\section{MATERIAL AND METHODS}

This is a cross-sectional, descriptive, retrospective study conducted in a Brazilian medium complexity university hospital.

\section{Place of study}

The University Hospital of the University of São Paulo (HU-USP) is a medium complexity hospital. With 259 beds, the hospital provides outpatient and emergency services to the local community of Butantã, a district of São Paulo city, and to the university community of USP (students, teachers and employees) and develops teaching and research activities in the healthcare field. The hospital has a Pediatric Emergency Room (PER) and an Adult Emergency Room (AER), in addition to 8 admission units: Clinical Surgery (CS), Medical Clinic (MC), Maternity (MAT), Nursery (NUR), Pediatric Clinic (PC), Adult Intensive Care Unit (AICU), Pediatric Intensive Care Unit (PICU) and Neonatal Intensive Care Unit (NICU).

\section{Dispensation of human albumin at HU-USP}

The HA dispensing by the HU-USP Pharmacy Division is subject to an institutional protocol that was implemented in 2004 and is based on ANVISA RDC No. 115, dated May 10, 2004 (Brasil, 2004). This institutional protocol classifies the HA therapeutic indications as presented in Table I and does not provide recommendations on dosage.

According to the institutional protocol of HU-USP, for HA to be dispensed by the Pharmacy, the prescriber must submit a request form for using it. This request consisted of a form where the prescriber presented the diagnosis, the therapeutic indication, the dosage regimen (dose, frequency, administration route and treatment time) proposed for the patient. In addition, the prescriber marked one of the indications presented in Table I. The request was then evaluated by pharmacists and, if necessary, it was submitted for approval of the hospital board of directors. Then, the HA dispensing was electronically registered.

\section{Inclusion and exclusion criteria}

We included all patients for who HA was requested between January 1, 2017 and October 31, 2017, regardless of age. Therefore, none patient would be excluded in analysis. 
The appropriate use of human albumin in a Brazilian University Hospital: therapeutic indication and dosage regimen

TABLE I - HA therapeutic indications according to the institutional protocol of HU-USP

\section{Unquestionable indications}

Treatment of massive ascites by repeated paracentesis

After evacuation paracentesis in massive ascites

Hepatic cirrhosis and nephrotic syndrome, when there is diuretic-refractory edema, that pose imminent risk to patients' lives (massive pleural or pericardial effusion or ascites)

\section{Debatable indications}

In patients with cirrhosis and prevalence of spontaneous bacterial peritonitis

Newborn hyperbilirubinemia due to peri-natal hemolytic disease Others

If this option is checked, the prescriber must provide a justification for the use of human albumin.

\section{Data collect}

The information was obtained from the HA dispensing electronic record of the Pharmacy Division, the HA request forms, the medical records and prescriptions of the patients who received HA within the period defined by the study.

The electronic record consisted of software developed by the Department of Informatics of HUUSP, from which it was possible to extract reports on the consumption of HA in the hospital. From this report we obtained the number of bottles dispensed by the Pharmacy and the list of patients for whom HA was dispensed.

The data collected from the HA request forms were: patient identification number, admission unit, diagnosis and indication that would justify the use of HA, the proposed dosage regimen and treatment time.

From the patient identification number obtained in the HA request forms, we made consultations at the Statistical Medical Archive Service (SMAS) archives to analyze the patient's records. The medical records were classified as unavailable if after 3 attempts in this sector they were not available for consultation. Patientrelated complementary information (age and weight) and clinical status were obtained from the patient's records. In addition, the HA prescriptions were analyzed to verify the dosage regimen of these patients.

\section{Analysis of the appropriate use of human albumin}

The analysis of the appropriate use of albumin was performed according to two criteria: therapeutic indication in accordance with institutional protocol - based on RDC
No. 115, dated May 10, 2004; indication and dosage regimen, according to HA clinical guidelines published in the last years $(2007-2017)$.

\section{Institutional protocol: therapeutic indication}

Knowing that the objective of this stage is to classify therapeutic indications presented in the HA request forms as "appropriate" or "inappropriate" in relation to the institutional protocol, the following strategy was adopted:

Initially, the therapeutic indications checked in the HA request forms were ranked according to the classification presented in Table I (unquestionable, debatable, and other indications). Indications marked "unquestionable" and "debatable" were classified as "appropriate" because they were indications where albumin using was supported by the institutional protocol. The indications marked "other" had their justifications analyzed because there could be a filling error. If the indication was described in the protocol, it would be classified as "appropriate"; in other situations, the indication was considered "inappropriate".

\section{Clinical protocols published in the last years: therapeutic indication and dosage regimen}

For this stage, a search was made to find clinical protocols that presented recommendations for the rational use of albumin. Pubmed database and Google $\mathbb{R}$ web search engine were used, applying the following descriptors: clinical practice guide, clinical protocol and albumin in English, Portuguese and Spanish languages. Only the documents published in the last years (2007 2017) were included in the analysis. From the findings, a table was drawn up with the synthesis of recommendations for each of the therapeutic indications of HA.

The use of HA was considered "appropriate" in those cases that the use reported in the medical record were in agreement with the recommendations found in the review. In cases that the conditions for the use of HA were recommended, the indication was considered appropriate only if the patient's condition met these criteria. The use was classified as "inappropriate" only in those situations that there was no recommendation or it was contrary to the recommendation. In the case of the dosage regimen analysis, dose and proposed treatment time were main criteria evaluated. In this stage of analysis, data of the request forms and medical records were considered.

\section{Ethical aspects}

The study was submitted to the Research Ethics Committee (REC) of the Faculty of Pharmaceutical 
Sciences of the University of São Paulo and the University Hospital of the University of São Paulo (CAAE: 65583017.8.3001.0076). Since the study offered minimal risk to the patients involved, the application of the Informed Consent Form (ICF) was dispensed.

\section{RESULTS AND DISCUSSION}

According to the pharmacy dispensing records, HA was dispensed for 46 patients between January and October 2017. The Pharmacy Division received 98 requests in this period, with the request average number per patient being $2.1 \pm 1.5$, ranging from 1 to 7 requests per patient. This variation was observed because some patients received HA on more than one occasion, which is also reported in other studies (Matos, Rozenfeld, 2005). In addition, for indications where HA needed to be given for more than one day (e.g., spontaneous bacterial peritonitis or hepatorenal syndrome), it was not uncommon to present a request form for each day that HA would be given. Perhaps a modification in the "treatment time" field to fill in the request form will minimize this misinterpretation.

In HU-USP, the use of HA was common among adults $(55 ; 56.1 \%)$ and pediatric patients $(43 ; 43.9 \%)$ as can be seen in Table II. The admission unit that most requested HA was PICU $(28 ; 28.6 \%)$, followed by MC $(24 ; 24.5 \%)$ and AER $(19 ; 19.4 \%)$. This distribution is not related to the results found in other studies that analyzed the appropriate use of HA in their hospitals. This difference occurred mainly from the fact that the studies found in the literature were performed in tertiary and quaternary hospitals, so that the units that most requested HA in these hospitals were specialized units such as gastroenterology, cardiology and transplantation (Casuccio et al., 1998, Borges Filho et al., 2010, Vargas et al., 1997).

\section{Analysis of the appropriate use of human albumin}

\section{Institutional protocol: therapeutic indication}

The indications marked in the 98 requests are presented in Table III.

From the results obtained in Table III it was possible to observe that $51(52.0 \%)$ of the 98 requests marked the option "other" when requesting HA for the Pharmacy Division, mainly among pediatric patients. Other Brazilian studies have also shown that HA is frequently requested for other indications not included in the national guidelines (Borges Filho et al., 2010; Matos, Rozenfeld, 2005). However, in these studies, the rate of unsubstantiated indications was $28 \%$ and $20.9 \%$, respectively, with small or none contribution of
TABLE II - Requests for human albumin, per admission unit (HU-USP, from January to October 2017)

\begin{tabular}{lcc}
\hline & Unit & N (\%) \\
\hline & MC & $24(24.5)$ \\
Adult & AER & $19(19.4)$ \\
( $\mathbf{1 5}$ years) & CS & $7(7.1)$ \\
& AICU & $5(5.1)$ \\
& All & $\mathbf{5 5 ( 5 6 . 1 )}$ \\
\hline & PICU & $28(28.6)$ \\
Pediatric & PC & $11(11.2)$ \\
(<15 years) & PER & $2(2.0)$ \\
& NICU & $2(2.0)$ \\
& All & $43(43.9)$ \\
\hline Total & & $\mathbf{9 8}(\mathbf{1 0 0 . 0})$ \\
\hline
\end{tabular}

MC - Medical Clinic; AER - Adult Emergency Room; CS Clinical Surgery; AICU - Adult Intensive Care Unit; PICU - Pediatric Intensive Care Unit; PC - Pediatric Clinic; PER Pediatric Emergency Room; NICU - Neonatal Intensive Care Unit.

pediatric patients. When only adult patients of the study are considered, the rate of unsubstantiated indications was $14(14.3 \%)$, becoming more similar to the rates found in the literature. Unfortunately, the scientific literature on the HA use in pediatric populations is scarce, which makes it impossible to compare them with the results found in the present study.

It is important to highlight that these 51 requests with the "other" option marked referred to 29 patients, of which 3 presented more than one request with different indications ("unquestionable" and "other") in a short period (maximum of 3 days). These 3 patients were pediatric and the "unquestionable" indication was "liver cirrhosis and nephrotic syndrome", when there is diureticrefractory edema, that pose imminent risk to patients' lives (massive pleural or pericardial effusion or ascites), while the indication "other" was related to "nephrotic syndrome". This "duplicity" of choice (between "unquestionable" and "other" indications) was also observed in the study by Matos and Rozenfeld (2005), but the cause of this "duplicity" was not presented. In the present study, what was observed is an apparent contradiction, since a single disease (nephrotic syndrome) was between the "unquestionable" and "other" indications. One possible explanation for this finding was the specificity of the criteria for the use of HA in nephrotic syndrome to be considered unquestionable. According to RDC No. 115, dated May 10, 2004, the use of HA for this indication is only recommended for cases in which "there are edemas 
The appropriate use of human albumin in a Brazilian University Hospital: therapeutic indication and dosage regimen

TABLE III - Indications marked in the request form for human albumin use (HU-USP, from January to October 2017)

\begin{tabular}{|c|c|c|c|c|}
\hline Indications & & $\begin{array}{l}\text { Adult } \\
\mathrm{N}(\%)\end{array}$ & $\begin{array}{l}\text { Pediatric } \\
\mathrm{N}(\%)\end{array}$ & $\mathrm{N}(\%)$ \\
\hline \multirow{4}{*}{ Unquestionable } & Treatment of massive ascites by repeated paracentesis & $3(3.1)$ & $0(0.0)$ & $3(3.1)$ \\
\hline & After evacuation paracentesis in massive ascites & $5(5.1)$ & $0(0.0)$ & $5(5.1)$ \\
\hline & $\begin{array}{l}\text { Hepatic cirrhosis and nephrotic syndrome, when there is diuretic- } \\
\text { refractory edema, that pose imminent risk to patients' lives (massive } \\
\text { pleural or pericardial effusion or ascites) }\end{array}$ & $13(13.3)$ & $5(5.1)$ & $18(18.4)$ \\
\hline & All & $21(21.5)$ & $5(5.1)$ & $26(26.6)$ \\
\hline \multirow{3}{*}{ Debatable } & $\begin{array}{l}\text { In patients with cirrhosis and prevalence of spontaneous bacterial } \\
\text { peritonitis }\end{array}$ & $18(18.4)$ & $0(0.0)$ & $18(18.4)$ \\
\hline & $\begin{array}{l}\text { Newborn hyperbilirubinemia due to peri-natal hemolytic disease } \\
\text { (HDN) }\end{array}$ & $0(0.0)$ & $0(0.0)$ & $0(0.0)$ \\
\hline & All & $18(18.4)$ & $0(0.0)$ & $18(18.4)$ \\
\hline \multirow{6}{*}{ Others } & Shock related (hypovolemic or septic) & $1(1.0)$ & $27(27.6)$ & $28(28.6)$ \\
\hline & Related to hepatorenal syndrome & $10(10.2)$ & $0(0.0)$ & $10(10.2)$ \\
\hline & Related to nephrotic syndrome & $0(0.0)$ & $8(8.2)$ & $8(8.2)$ \\
\hline & Related to anuria or oliguria & $2(2.1)$ & $1(1.0)$ & $3(3.1)$ \\
\hline & Related to edema or anasarca & $1(1.0)$ & $1(1.0)$ & $2(2.0)$ \\
\hline & All & $14(14.3)$ & $37(37.7)$ & $51(52.0)$ \\
\hline Unregistered & & $2(2.1)$ & $1(1.0)$ & $3(3.1)$ \\
\hline Total & & $55(56.1)$ & $43(43.9)$ & $98(100)$ \\
\hline
\end{tabular}

that are refractory to diuretics and pose an imminent risk to patients' lives (massive pleural effusions or pericardial or ascites)". Therefore, for those patients that treatment was not refractory to diuretics or, in the prescriber's evaluation was not characterized as a condition of imminent risk to patient's life, the use of HA was not recommended, being included in the option "other ".

Therefore, due to the impossibility of verifying if the requests whose "other" option was marked and the justification related to the nephrotic syndrome respected the condition of refractoriness or life-threatening, the indication of HA for these requests was considered "inappropriate". In addition, none of the other justifications could be supported by the protocol, and therefore all requests with the "other" option marked were considered "inappropriate". Requests classified as "unregistered", that is, those that had no indication marked in the request were also considered inappropriate. The final result of this classification, with the analysis of the appropriate indication of the HA according to the institutional protocol is presented in Table IV.

Clinical protocols published in the last years: therapeutic indication and dose regimen

The synthesis of the recommendations was performed with recommendations of five clinical protocols
TABLE IV - Analysis of the appropriate therapeutic indications of human albumin according to the institutional protocol (HUUSP, from January to October 2017)

\begin{tabular}{lc}
\hline Indications & N (\%) \\
\hline $\begin{array}{l}\text { Appropriate in relation to the institutional } \\
\text { protocol }\end{array}$ & $44(44.9)$ \\
$\begin{array}{l}\text { Inappropriate in relation to the institutional } \\
\text { protocol }\end{array}$ & $54(55.1)$ \\
\hline Total & $\mathbf{9 8 ( 1 0 0 )}$ \\
\hline
\end{tabular}

and is shown in Table V. Only the therapeutic indications in the requests of using HA in HU-USP are presented.

When searching medical records of the 46 patients who received HA during the study period, only 25 (54.3\%) were available for consultation at the SMAS. Therefore, at this stage, the analysis was limited to 25 patients, resulting in 54 forms.

Analyzing the recommendations of the synthesis table, it was observed that, for some indications, the use of HA was conditioned. For example, according to the summary table the use of HA in cases of spontaneous bacterial peritonitis should be associated with the antibiotics use. Table VI presents these conditions of using. 
Table VII presents the comparison between the information found in the medical records and the presented conditions and the table of synthesis (Table V).

In other words, from Table VII it was possible to affirm that the conditions presented in Table VI were followed in all cases in which the HA use was indicated. This suitability rate was higher than that observed in other studies of the appropriate use of HA. The study

TABLE V - Synthesis of recommendations for human albumin use

\begin{tabular}{ll}
\hline Indication & Recommendation \\
\hline & Small volume $(<4$ or 5 liters) - Use crystalloids as \\
& first choice (University Hospital Consortium, 2010). \\
& If management of condition is refractory to sodium \\
& restriction $(90 \mathrm{mmol} /$ day), HA may be considered \\
& (Liumbruno et al., 2009). In patients with cirrhosis: \\
& HA may be indicated as option if there are concerns \\
& regarding use of synthetic colloids or crystalloids \\
& (volume overload, renal failure, coagulopathy) \\
& (Stanford Health Care, 2017; Guy's and St. Thomas' \\
& NHS Foundation Trust, 2015; Caraceni et al., 2016).
\end{tabular}

Paracentesis

\section{Hepatorenal Syndrome (HRS)}

Large volume ( $\geq 4$ or 5 liters $)$ - There is widespread consensus on the HA use (Vermeulen, Ratko, Erstad, 2010; Liumbruno et al., 2009). In patients with cirrhosis: Should be use HA and avoid combinations with other plasma expanders to reduce the dose of HA. Consider oral midodrine or terlipressin, mainly in critical care (Guy's and St. Thomas' NHS Foundation Trust, 2015; Caraceni et al., 2016; National Plasma Products Expert Advisory Group - Scotland NHS, 2016; Ontario Regional Blood Coordinating Network, 2012).

For diagnosis of Suspected ${ }^{\mathbf{A}}-$ HA may benefit. Diuretics use should be discontinued and considered volume expansion with HA for 2 consecutive days, then it should be evaluated if there was improvement of renal function (Vermeulen, Ratko, Erstad, 2010; Liumbruno et al., 2009; Stanford Health Care, 2017). In patients with cirrhosis: HA is used regularly (Caraceni et al., 2016).

Confirmed $^{\mathrm{B}}$ - HA may be clinically indicated after 72 hours, nephrology and hepatology services should be consulted on the maintenance of the HA use. Should be used with vasoconstricting drugs (midodrine and octreotide) (Vermeulen, Ratko, Erstad, 2010; Liumbruno et al., 2009; Stanford Health Care, 2017; National Plasma Products Expert Advisory Group - Scotland NHS, 2016; Lyu et al.; 2016). Eligible for liver transplantation: HA should be used with vasoactive drugs, consider terlipressin (Guy's and St. Thomas' NHS Foundation Trust, 2015). In patients with cirrhosis: HA should be given with other vasoconstrictors (noradrenaline or midodrine plus octreotide) (Caraceni et al., 2016).
Dosing recommendation

$20 \mathrm{~g}$ of albumin $20 \%$ per every $3 \mathrm{~L}$ ascites drained or $20 \mathrm{~g}$ of albumin per every $2 \mathrm{~L}$ if renal function impaired (Guy's and St. Thomas' NHS Foundation Trust, 2015; National Plasma Products Expert Advisory Group - Scotland NHS, 2016).

6 to $8 \mathrm{~g}$ of albumin $25 \%$ per every liter ascitic fluid removed (Vermeulen, Ratko, Erstad, 2010; Liumbruno et al., 2009; Stanford Health Care, 2017; Caraceni et al., 2016).

Suspected - $1 \mathrm{~g} / \mathrm{kg}$ of albumin $25 \%$ (up to $100 \mathrm{~g}$ ) daily for 2 consecutive days (Vermeulen, Ratko, Erstad, 2010; Stanford Health Care, 2017; Guy's and St. Thomas' NHS Foundation Trust, 2015; Caraceni et al., 2016).

Confirmed - Day 1: $1 \mathrm{~g} / \mathrm{kg}$ of albumin $5 \%$ or $20 \%$

Day 2 to 16: 20 to $40 \mathrm{~g}$ of albumin $5 \%$ or $20 \%$ daily (Vermeulen, Ratko, Erstad, 2010; National Plasma Products Expert Advisory Group Scotland NHS, 2016)

Day 1: $1 \mathrm{~g} / \mathrm{kg}$ of albumin $20 \%$ or $25 \%$

Day 2 to $14: 0.5 \mathrm{~g} / \mathrm{kg}$ of albumin $20 \%$ or $25 \%$ daily (Guy's and St. Thomas' NHS Foundation Trust, 2015; Ontario Regional Blood Coordinating Network, 2012).

Albumin should be given with vasoconstrictors (Caraceni et al., 2016; National Plasma Products Expert Advisory Group - Scotland NHS, 2016). 
TABLE V - Synthesis of recommendations for human albumin use (cont.)

\begin{tabular}{l}
\hline Indication \\
\hline \\
Spontaneous Bacterial \\
Peritonitis (SBP)
\end{tabular}

Recommendation

HA according clinical conditions (including patients with cirrhosis) (Vermeulen, Ratko, Erstad, 2010; Stanford Health Care, 2017; Guy's and St. Thomas' NHS Foundation Trust, 2015; National Plasma Products Expert Advisory Group - Scotland NHS, 2016; Ontario Regional Blood Coordinating Network, 2012).

Low-risk patients ${ }^{\mathrm{C}}$ - decision on use of HA should be individualised; HA use could be restricted to "high" risk patients.

High-risk patients - HA administration is mandatory for patients with cirrhosis and is not recommended Dosing recommendation Peritonitis (SBP) the use of crystalloids and synthetic colloids or association these with HA (Caraceni et al., 2016; National Plasma Products Expert Advisory Group Scotland NHS, 2016).

HA may be considered in cases with severe refractory edema or symptoms and signs of sub-acute intravascular volume depletion, including oliguria,

Severe Nephrotic Syndrome poor peripheral perfusion, abdominal pain, raised creatinine and haematocrit; always under supervision of consultant paediatric nephrologist (Guy's and St. Thomas' NHS Foundation Trust, 2015).

Crystalloids should be considered first-line therapy (Vermeulen, Ratko, Erstad, 2010; National Plasma Products Expert Advisory Group - Scotland NHS, 2016). Inappropriate use of HA (Stanford Health Care, 2017). Maybe used in patients with refractory septic shock who have received resuscitation with crystalloid and require $>0.2 \mathrm{mcg} / \mathrm{kg} / \mathrm{min}$ of norepinephrine (Guy's and St. Thomas' NHS Foundation Trust, 2015). Consider use of HA in critical care (Lyu et al., 2016).

Maldistributive shock
Day 1: $1.5 \mathrm{~g} / \mathrm{kg}$ of albumin $5 \%, 20 \%$ or $25 \%$. Day $3: 1 \mathrm{~g} / \mathrm{kg}$ of albumin $5 \%, 20 \%$ or $25 \%$. (Caraceni et al., 2016; National Plasma Products Expert Advisory Group - Scotland NHS, 2016).

Albumin should be given in association with antibiotics (Guy's and St. Thomas' NHS Foundation Trust, 2015).
$1 \mathrm{~g} / \mathrm{kg}$ of albumin $20 \%$ over 6 hours, with or without loop diuretic (Guy's and St. Thomas' NHS Foundation Trust, 2015).

Adults: 20 to $40 \mathrm{~g}$ of albumin over 30 to 60 minutes (Guy's and St. Thomas' NHS Foundation Trust, 2015).

Children: Without evidence.

A-Suspected HRS: serum creatinine $>1.5 \mathrm{mg} / \mathrm{dL}$ in the presence of cirrhosis, B - Confirmed HRS: i. serum creatinine $>1.5 \mathrm{mg} / \mathrm{dL}$ in the presence of cirrhosis; ii. absence of shock, ongoing bacterial infection, and/or current treatment with nephrotoxic drugs; iii. absence of sustained improvement in renal function after discontinuation of diuretics and a trial of albumin $1 \mathrm{~g} / \mathrm{kg}$; iv. absence of proteinuria $(<500 \mathrm{mg}$ /day) or hematuria $(<50$ red cells per high-power field); $\mathrm{v}$. absence of ultrasonographic evidence of obstructive uropathy or parenchymal renal disease. C - Low risk patients: baseline serum bilirubin $<4 \mathrm{mg} / \mathrm{dL}$ and serum creatinine $<1 \mathrm{mg} / \mathrm{dL}^{3}$.

TABLE VI - Conditions for the use of HA according to other clinical protocols

\begin{tabular}{cc}
\hline Indication & Recommendation \\
\hline Paracentesis & Volume of ascites fluid drained $(>4 \mathrm{~L})$ \\
Hepatorenal Syndrome & Without evaluation criteria \\
Spontaneous Bacterial Peritonitis (SBP) & Association with antibiotics \\
Nephrotic syndrome in children & Refractory condition to diuretic use \\
Maldistributive shock & Refractory condition to crystalloid use \\
\hline
\end{tabular}


whose numbers approached most was an Italian study conducted in October 2013. According to this study, the indication suitability was observed in $83.3 \%$ of the requests (Casuccio et al., 2015).

TABLE VIII - Analysis of dosage regimens suitability, according to other clinical protocols (HU-USP, from January to October 2017)

\begin{tabular}{lc}
\hline Dosage regimen & N (\%) \\
\hline Appropriate & $13(24.1)$ \\
Maldistributive shock & $9(16.7)$ \\
Nephrotic syndrome in children & $8(14.8)$ \\
Spontaneous bacterial peritonitis (SBP) & $5(9.2)$ \\
High volume paracentesis & $3(5.5)$ \\
Suspicion of hepatorenal syndrome & $\mathbf{3 8 ( 7 0 . 3 )}$ \\
All & \\
\hline Inappropriate & $4(7.4)$ \\
Spontaneous bacterial peritonitis (SBP) & $4(7.4)$ \\
Suspicion of hepatorenal syndrome & $3(5.6)$ \\
Maldistributive shock & $3(5.6)$ \\
Nephrotic syndrome in children & $2(3.7)$ \\
No indication of use & $0(0.0)$ \\
High volume paracentesis & $\mathbf{1 6 ( 2 9 . 7 )}$ \\
All & \\
\hline Total & $\mathbf{5 4 ( 1 0 0 )}$ \\
\hline
\end{tabular}

When comparing the suitability rate of the therapeutic indications, according to the institutional protocol (Table IV), with the suitability rate of the therapeutic indications, according to other clinical protocols (Table VII), it is possible to observe a great divergence between the two suitability profiles. According to the RDC $\mathrm{n}^{\mathrm{o}} 115$, the use of HA is considered inappropriate for maldistributive shock (volemic or septic) and hepatorenal syndrome. In the case of shock, the use of HA is still much debated in clinical practice, so its use is not yet recommended in many clinical protocols. This is because the use of HA as a volume expander in critically ill patients in septic shock is considered safe (Caraceni et al., 2015). However, when comparing the use of colloids (HA) and crystalloids as volume expander, no superiority was found in relation to the effectiveness of the colloid on the crystalloid, so that the crystalloid is recommended as a first choice solution because its lower cost and lower rate of adverse effects (Medeiros et al., 2015). However, in the case of hepatorenal syndrome, the use of HA is already considered a formal indication (Valerio et al., 2016; Bernardi, Ricci,
Zaccherini, 2014). Thus, from the results obtained, it is possible to affirm that an updating of the institutional protocol is necessary, including septic shock as a condition in which HA is adequately indicated when the patient is refractory to crystalloids, and hepatorenal syndrome as an appropriate indication therapy for the use of HA.

Regarding the dosage regimen, Table VIII presents the comparison between those found in medical records and the recommended in Table VI.

Different from the appropriateness of the indication profile presented in Table VIII, when the dosage regimen was analyzed, inappropriate use was observed in $16(29.7 \%)$ requests. When searching for studies to compare the profile found, it was observed that, despite the inappropriate use of HA is widely discussed in the literature, no study has yet evaluated the types found. In the present study, inadequate dosage varied mainly in relation to the HA prescribed dose and the treatment time. In addition, in the case of septic shock, no guideline studied showed a definite dosage for the use of HA in children. However, databases such as Micromedex ${ }^{\circledR}$ and Lexicomp ${ }^{\circledR}$, and references such as the Blackbook Pediatria, indicated the use of HA for this condition. The most frequently found dose was $1 \mathrm{~g} / \mathrm{kg}$. Given that the institutional protocol does not show dosage schedules in the HA request form, the present study proposes that when updating this protocol, the dosage regimens must be included.

TABLE VII - Analysis of the appropriateness of therapeutic indications, according to other clinical protocols (HU-USP, from January to October 2017)

\begin{tabular}{lc}
\hline Indications & N (\%) \\
\hline Appropriate & $16(29.6)$ \\
$\quad$ Maldistributive shock & $12(22.2)$ \\
Nephrotic syndrome in children & $12(22.2)$ \\
Spontaneous bacterial peritonitis (SBP) & $7(13.0)$ \\
Suspicion of hepatorenal syndrome & $5(9.3)$ \\
$\quad$ High volume paracentesis & \\
\hline Inappropriate & $2(3.7)$ \\
$\quad$ No indication of use & $\mathbf{5 4 ( 1 0 0 )}$ \\
\hline Total
\end{tabular}

\section{Limitations and strengths of the study}

Regarding the study limitations, it was performed in a university medium complexity hospital where the use of HA should not be very frequent. Thus, the profile 
of HA use in the institution can be very particular, not being similar to the profile of use found in the literature. In addition, the review to seek information on albumin use in clinical protocols was not performed in a systematic manner, with evaluation of the documents quality. Finally, the unavailability of almost $50 \%$ of patient's records reduced the sample size and may have affected the suitability rate of the therapeutic indications.

On the other hand, the question of the rational use of albumin is still current and there are few studies of HA that include pediatric patients or the suitability of dose regimens. In addition, the fact that compliance with other clinical protocols is much higher than when comparing with the institutional protocol shows that prescribers are up to date with the evidence regarding the use of HA, which is expected in a university hospital. They are not linking their practice to the RDC, possibly because that document was published over 10 years ago and certainly needs to be updated.

\section{CONCLUSION}

The present study evaluated the appropriateness of the use of HA in relation to an institutional protocol based on Brazilian regulations and other clinical guidelines. We observed lack of updating of the institutional protocol, since it has been shown to play a fundamental role in the results observed as inappropriate. The major problem in $\mathrm{HU} / \mathrm{USP}$ was the dose regimen. In addition, the University Hospital of the University of São Paulo (HU-USP) is a secondary hospital, and the use of HA should not be so frequent in the institution, since the studies presented on the use of HA report the use in high complexity hospitals (tertiary and quaternary), with specialties such as gastroenterology, cardiology and transplantation as the main requesters for HA in these institutions.

\section{ACKNOWLEDGEMENT}

The authors would like to express their gratitude to the Ministry of Health and to the Ministry of Education for the financial support provided for this study and for the Pharmacy Residency Program of which one of the authors participates.

\section{REFERENCES}

Bernardi M, Ricci CS, Zaccherini G. Role of human albumin in the management of complications of liver cirrhosis. J Clin Exp Hepatol. 2014;4(4):302-11.
Borges Filho WM, Almeida SM de, Ferracini FT, Fernandes Júnior CJ. Pharmacy contribution to the prescription and rational use of human albumin at a large hospital. Einstein (São Paulo). 2010;8(2):215-20.

Brasil. Ministério da Saúde. Agência Nacional de Vigilância Sanitária. Resolução RDC no 115, de 10 de maio de 2004. Aprova as Diretrizes para o uso de Albumina. Diário Oficial da União, Brasília, DF, 11 maio 2004. [Internet] 2004. [cited 2017 Dec 29] Available from: <http://http://redsang.ial.sp.gov.br/site/ docs_leis/rs/rs12.pdf $>$

Caraceni P, Domenicali M, Tovoli A, Napoli L, Ricci CS, Tufoni M, et al. Clinical indications for the albumin use: Still a controversial issue. Eur J Intern Med. 2013;24(8):721-8.

Caraceni P, Tufoni M, Bonavita ME. Clinical use of albumin. Blood Transfus. 2013;11(Suppl 4):s18-s25.

Caraceni P, Angeli P, Prati D, Bernardi M, Alessandria C, Riggio O, et al. AISF-SIMTI Position Paper: The appropriate use of albumin in patients with liver cirrhosis. Dig Liver Dis. 2016 Jan;48(1):4-15.

Casuccio A, Nalbone E, Immordino P, Seta C La, Sanfilippo P, Tuttolomondo A, et al. Appropriateness of requests for human serum albumin at the University Hospital of Palermo, Italy: A prospective study. Int J Qual Heal Care. 2015;27(2):154-60.

Corrêa TD, Rocha LL, Pessoa CMS, Silva E, Assunção MSC de. Fluid therapy for septic shock resuscitation: which fluid should be used? Einstein (São Paulo). 2015;13(3):462-8.

Guy's and St. Thomas' NHS Foundation Trust. Guideline for the use of human albumin solution (HAS). 2015. Available on: https://www.sps.nhs.uk/wp-content/uploads/2015/05/ Guideline20use20human20albumin20solution20and20 demand20management20final.pdf

Liumbruno GM, Bennardello F, Lattanzio A, Piccoli P, Rossetti G. Recommendations for the use of albumin and immunoglobulins. Blood Transfus. 2009;7(3):216-34.

Lyu PF, Hockenberry JM, Gaydos LM, Howard DH, Buchman TG, Murphy DJ. Impact of a Sequential Intervention on Albumin Utilization in Critical Care. Crit Care Med. 2016;44(7):1307-13.

Matos GC de, Rozenfeld S. Avaliação do uso de albumina humana em hospital do Rio de Janeiro, Brasil. Cad Saúde Pública. 2005;21(4):1224-33. 
Medeiros DNM, Ferranti JF, Delgado AF, Carvalho WB de. Colloids for the initial management of severe sepsis and septic shock in pediatric patients: a systematic review. Pediatr Emerg Care. 2015;31(11):e11-6.

National Plasma Products Expert Advisory Group - Scotland NHS. Clinical guidelines for human albumin use. 2016. Available on: http://www.nsd.scot.nhs.uk/documents/ guidelines/albumingdl.pdf.

Ontario Regional Blood Coordinating Network. Ontario Albumin Administration Recommendations. 2012. Available on: http://www.nsd.scot.nhs.uk/documents/guidelines/albumingdl. pdf.

Stanford Health Care. Guidelines for Intravenous Albumin Administration at Stanford Health Care. 2017. Available on: https://stanfordhealthcare.org/content/dam/SHC/healthcare-professionals/medical-staff/medstaff-weekly/20170315guidelines-for-intravenous-albumin-administration.pdf

Vermeulen LC Jr, Ratko TA, Erstad BL. A paradigm for consensus: The University Hospital Consortium guidelines for the use of albumin, nonprotein colloid, and crystalloid solutions. Arch Intern Med. 1995 (revised 2005 \& 2010);155:373-379. Available on: https://www.universityhealthsystem.com/ / media/files/clinical-pathways/albumin-guidelines-0410.pdf.
Tullis JL. Albumin. 1. Background and use. JAMA. $1977 \mathrm{a} ; 237(4): 355-60$

Tullis JL. Albumin. 2. Guidelines for clinical use. JAMA. 1977b;237(5):460-3

Vaglio S, Calizzani G, Grazzini G, Lanzoni M, Liumbruno GM. Italian albumin usage (or misusage?). Eur J Intern Med. 2014;25(3):e31-2

Valerio C, Theocharidou E, Davenport A, Agarwal B. Human albumin solution for patients with cirrhosis and acute on chronic liver failure: Beyond simple volume expansion. World J Hepatol. 2016;8(7):345-54.

Vargas E, De Miguel V, Portolés A, Avendaño C, Ambit MI, Torralba A, et al. Use of albumin in two Spanish university hospitals. Eur J Clin Pharmacol. 1997;52(6):465-70.

Received for publication on $05^{\text {th }}$ January 2018 Accepted for publication on $15^{\text {th }}$ March 2018 